Research Article

\title{
Classical Theory of Linear Multistep Methods for Volterra Functional Differential Equations
}

\author{
Yunfei $\mathrm{Li}\left(\mathrm{DD}^{1}\right.$ and Shoufu $\mathrm{Li}^{2}$ \\ ${ }^{1}$ College of Mathematics and Physics, Hunan Province Cooperative Innovation Center for the Construction and Development of \\ Dongting Lake Ecological Economic Zone, Hunan University of Arts and Science, Changde 415000, Hunan, China \\ ${ }^{2}$ Department of Mathematics, Xiangtan University, Xiangtan 411105, China \\ Correspondence should be addressed to Yunfei Li; feiyun2cc@163.com
}

Received 5 November 2020; Accepted 23 December 2020; Published 12 March 2021

Academic Editor: Piergiulio Tempesta

Copyright ( $) 2021$ Yunfei Li and Shoufu Li. This is an open access article distributed under the Creative Commons Attribution License, which permits unrestricted use, distribution, and reproduction in any medium, provided the original work is properly cited.

Based on the linear multistep methods for ordinary differential equations (ODEs) and the canonical interpolation theory that was presented by Shoufu Li who is exactly the second author of this paper, we propose the linear multistep methods for general Volterra functional differential equations (VFDEs) and build the classical stability, consistency, and convergence theories of the methods. The methods and theories presented in this paper are applicable to nonneutral, nonstiff, and nonlinear initial value problems in ODEs, Volterra delay differential equations (VDDEs), Volterra integro-differential equations (VIDEs), Volterra delay integro-differential equations (VDIDEs), etc. At last, some numerical experiments verify the correctness of our theories.

\section{Introduction}

VFDEs contain many subtypes, such as VDDEs, VIDEs, VDIDEs, etc. Certainly ODEs are also a subtype of them. VFDEs are widely applied in many fields of science and technology (see [1-5] and their references), for which there have been remarkable theoretical and numerical analysis research results. As for VDDEs, please refer to [6-19], as for VIDEs, please refer to [20-23], and as for VDIDEs, please refer to [24-29]. In recent decades, Li [30-35] has carried on systematic research for stiff general VFDEs and the numerical methods for them. In 2014, Li [36] established the classical stability and convergence theories of Runge-Kutta methods for nonstiff, nonlinear general VFDEs. It is well known that in solving ODEs, linear multistep methods have significant advantages in the aspects of format simplification and computation cost, and experts have presented many famous linear multistep methods such as Backward Differentiation Formula (BDF) methods and Adams methods. Based on the linear multistep methods for ODEs and the canonical interpolation theory presented by $\mathrm{Li}$ [34], we propose the linear multistep methods for general VFDEs and build the classical stability, consistency, and convergence theories of the methods. The methods and theories presented in this paper are applicable to nonneutral, nonstiff, and nonlinear initial value problems in ODEs, VDDEs, VIDEs, VDIDEs, etc., and are interesting companions to the methods and theories in [36]. Furthermore, the paper may have some value for the study of numerical methods for fractional order VFDEs because many numerical methods for fractional-order differential equations are presented by extending the methods for integer-order differential equations.

This paper is organized as follows. In Section 2, the linear multistep methods for general VFDEs are introduced. The classical stability of the linear multistep methods is discussed in Section 3. The classical consistency and convergence analyses are carried out in Section 4. Some numerical experiments are carried out in Section 5, which verifies the correctness of the theories presented in this paper.

\section{Derivation of the Numerical Methods}

Let $\mathbf{R}^{m}$ be the $m$-dimensional Euclidean space with standard inner product $\langle\cdot, \cdot\rangle$ and the corresponding norm $\|\cdot\|$. For any given closed interval $\mathbf{I} \subset \mathbf{R}$, let $\mathbf{C}_{m}(\mathbf{I})$ denote the Banach space consisting of all continuous mappings $x: \mathbf{I} \longrightarrow \mathbf{R}^{m}$ with the maximum norm $\|x\|=\max _{t \in \mathrm{I}}\|x(t)\|$. 
Consider VFDEs of the form [30-32, 34, 36]

$$
\left\{\begin{array}{l}
y^{\prime}(t)=f(t, y(t), y(\cdot)), \quad t \in[0, T], \\
y(t)=\varphi(t), \quad t \in[-\tau, 0]
\end{array}\right.
$$

where $T \in(0,+\infty)$ and $\tau \in[0,+\infty]$ are constants, $\varphi(t) \in \mathbf{C}_{m}[-\tau, 0]$ is an initial function, and $f:[0, T] \times \mathbf{R}^{m} \times$ $\mathbf{C}_{m}[-\tau, T] \longrightarrow \mathbf{R}^{m}$ is a given continuous mapping which satisfies the classical Lipschitz conditions [36]:

$$
\left\{\begin{array}{l}
\left\|f\left(t, u_{1}, \psi(\cdot)\right)-f\left(t, u_{2}, \psi(\cdot)\right)\right\| \leq L_{1}\left\|u_{1}-u_{2}\right\|, \\
\forall t \in[0, T], u_{1}, u_{2} \in \mathbf{R}^{m}, \psi \in \mathbf{C}_{m}[-\tau, T], \\
\left\|f\left(t, u, \psi_{1}(\cdot)\right)-f\left(t, u, \psi_{2}(\cdot)\right)\right\| \leq L_{2} \max _{\xi \in[-\tau, t]}\left\|\psi_{1}(\xi)-\psi_{2}(\xi)\right\|, \\
\forall t \in[0, T], u \in \mathbf{R}^{m}, \psi_{1}, \psi_{2} \in \mathbf{C}_{m}[-\tau, T],
\end{array}\right.
$$

where $L_{1}$ and $L_{2}$ are classical Lipschitz constants. Equations (1) has a unique solution $y(t)$ [37], and we further make the same assumption as [36] that the system $y^{\prime}(t)=f(t, y(t), y(\cdot))(t \in[0, T])$ in (1) is stable for the initial function $\varphi(t) \in \mathbf{C}_{m}[-\tau, 0]$, which is to say there exists a function $C(t)>0(t \in[0, T])$ which satisfies $\max _{t \in[0, T]} C(t)$ has only moderate size such that

$$
\|y(t)-z(t)\| \leq C(t) \max _{\xi \in[-\tau, 0]}\|\varphi(\xi)-\chi(\xi)\|, \quad \forall t \in[0, T]
$$

where $z(t)$ is the solution of the perturbed problem:

$$
\left\{\begin{array}{l}
z^{\prime}(t)=f(t, z(t), z(\cdot)), \quad t \in[0, T] \\
z(t)=\chi(t), \quad t \in[-\tau, 0]
\end{array}\right.
$$

We use $\mathbb{D}\left(L_{1}, L_{2}\right)$ to denote the problem class consisting of all the equations of form (1) which satisfy condition (2) and all the other assumption conditions made above. The problem class $\mathbb{D}\left(L_{1}, L_{2}\right)$ contains nonneutral initial value problems in ODEs, DDEs, IDEs, DIDEs, etc. [36].

Combine the linear $k$-step method:

$$
\sum_{i=0}^{k} \alpha_{i} y_{n+i}=h \sum_{i=0}^{k} \beta_{i} f\left(t_{n+i}, y_{n+i}\right)
$$

for ODEs with piecewise Lagrangian interpolation operators $\Pi_{n i}^{h}$ which are constructed based on canonical interpolation theory $[34,36]$, and we get the linear $k$-step method:

$$
\left\{\begin{array}{l}
y_{n i}^{h}(t)=\Pi_{n i}^{h}\left(t ; \psi, y_{1}, y_{2}, \ldots, y_{n+k}\right), \quad-\tau \leq t \leq t_{n+i}, i=0,1, \ldots, k \\
\sum_{i=0}^{k} \alpha_{i} y_{n+i}=h \sum_{i=0}^{k} \beta_{i} f\left(t_{n+i}, y_{n+i}, y_{n i}^{h}(\cdot)\right)
\end{array}\right.
$$

for the VFDEs (1), where $k \geq 1$, all $\alpha_{i}$ and $\beta_{i}$ are real constants, $\alpha_{k} \neq 0,\left|\alpha_{0}\right|+\left|\beta_{0}\right|>0, \rho(\xi)=\sum_{i=0}^{k} \alpha_{i} \xi^{i}$ is called the characteristic polynomial of the method $(6), h=(T / N)$ is the stepsize, $N$ is a given appropriate positive integer, $t_{n}=$ $n h(n=0,1, \ldots, N)$ are grid points, $\psi \in \mathbf{C}_{m}[-\tau, 0]$ is an approximation to the initial function $\varphi(t), y_{0}:=\psi(0)$, $y_{n} \in \mathbf{R}^{m}$ is an approximation to the true value $y\left(t_{n}\right)$, and the piecewise interpolation operators $\Pi_{n i}^{h}: \quad \mathbf{R} \times \mathbf{C}_{m}$ $[-\tau, 0] \times \mathbf{R}^{m(n+k)} \longrightarrow \mathbf{C}_{m}\left[-\tau, t_{n+i}\right]$ based on $p$-degree piecewise Lagrangian interpolation polynomials are defined as follows: if $p=0$,

$$
\Pi_{n i}^{h}\left(t ; \psi, y_{1}, y_{2}, \ldots, y_{n+k}\right)=\left\{\begin{array}{l}
\psi(t), \quad t \in[-\tau, 0] \\
y_{j}, \quad t \in\left(t_{j-1}, t_{j}\right], j=1,2, \ldots, n+i,
\end{array}\right.
$$

and if $p \geq 1$,

$$
\Pi_{n i}^{h}\left(t ; \psi, y_{1}, y_{2}, \ldots, y_{n+k}\right)= \begin{cases}\psi(t), & t \in[-\tau, 0] \\ L_{j, p}(t), & t \in\left(t_{j-1}, t_{j}\right], j=1,2, \ldots, n+i\end{cases}
$$


with

$$
\left\{\begin{array}{l}
L_{j, p}(t)=\sum_{v=0}^{p} l_{q+v}(t) y_{q+v}, \quad q=j-\bar{j}, j=1,2, \ldots, n+i, \\
l_{q+v}(t)=\prod_{s=0, s \neq v}^{p} \frac{t-t_{q+s}}{t_{q+v}-t_{q+s}}, \quad q=j-\bar{j}, v=0,1, \ldots, p, j=1,2, \ldots, n+i,
\end{array}\right.
$$

where the integer $\bar{j}$ can be freely determined under the conditions

$$
1 \leq \bar{j} \leq j, \quad j+p-n-k \leq \bar{j} \leq p .
$$

According to $[34,36]$, we know a piecewise interpolation operator $\Pi_{n i}^{h}$ defined by (7) or by (8) and (9) which satisfies the canonical condition

$$
\left\{\begin{array}{l}
\max _{-\tau \leq t \leq t_{n+i}}\left\|\Pi_{n i}^{h}\left(t ; \psi, y_{1}, y_{2}, \ldots, y_{n+k}\right)-\Pi_{n i}^{h}\left(t ; \chi, z_{1}, z_{2}, \ldots, z_{n+k}\right)\right\| \leq c_{\pi} \max \left\{\max _{1 \leq j \leq n+k}\left\|y_{j}-z_{j}\right\|, \max _{-\tau \leq t \leq 0}\|\psi(t)-\chi(t)\|\right\}, \\
\forall \psi, \chi \in \mathbf{C}_{m}[-\tau, 0], y_{j}, z_{j} \in \mathbf{R}^{m}, j=1,2, \ldots, n+k,
\end{array}\right.
$$

where the constant $c_{\pi} \geq 1$ only depends on the polynomial order $p$ and does not depend on $n, h, y_{j}, z_{j}, \psi$ and $\chi$. In this paper, we always assume that $c_{\pi}$ is of moderate size. In fact, Li $[34,36]$ has given the computational formula of $c_{\pi}$ and found $c_{\pi}$, respectively, equals $1,1,1.250$, and 1.631 when $p$,

$$
y_{n i}^{h}(t)=\Pi_{n i}^{h}\left(t ; \psi, y_{1}, y_{2}, \ldots, y_{n+k-1}\right), \quad-\tau \leq t \leq t_{n+i}, i=0,1, \ldots, k-1,
$$

and now the method is an explicit linear $k$-step method which is a special case of (6).

In particular, we point out that when the linear $k$-step method (6) for VFDEs starts, on the one hand, like method (5) for ODEs, it needs $k$ starting values, and on the other hand, it may need several grid points as interpolation nodes to construct interpolation operator $\Pi_{n i}^{h}$. Based on the above statements, in this paper, we always assume the $k-1(k \geq t k)$ values $y_{1}, y_{2}, \ldots, y_{k-1}$, which are called additional starting values, should be provided in advance by other ways, and method (6) starts with $n=k-k \geq 0$. By the Banach contraction mapping principle, we conclude that, for any given starting data $\psi \in \mathbf{C}_{m}[-\tau, 0], y_{1}, y_{2}, \ldots, y_{\breve{k-1}} \in \mathbf{R}^{m}$, method (6) can uniquely determine the sequence $\left\{y_{n}, n=\breve{k}, \breve{k}+1, \ldots, N\right\}$ when $h \leq h_{\varphi}$, where $h_{\varphi}$ can be any number under the condition

$$
0<h_{\varphi}<\frac{\left|\alpha_{k}\right|}{\left|\beta_{k}\right|\left(L_{1}+c_{\pi} L_{2}\right)} .
$$

\section{Stability Analysis}

Firstly, we list some concepts and elementary facts about scalar linear difference equations as follows $[35,38,39]$. respectively, equals $0,1,2$, and 3 . We call method (5) as the mother method of method (6).

If $\beta_{k}=0$ in method (6), in order to reduce the amount of calculation, we define

Consider the $k$ th order scalar linear difference equation with constant coefficients:

$$
\sum_{j=0}^{k} a_{j} \bar{y}_{m+j}=b_{m}, \quad m=q_{0}, q_{0}+1, \ldots
$$

here and later, $q_{0} \geq 0$ is an integer constant, and where $k \geq 1$ is an integer constant, every $a_{j}$ is a complex constant, $a_{k} \neq 0$, any $b_{m}$ is a complex number depending on $m$. If a complex number sequence $\bar{y}_{q_{0}}, \bar{y}_{q_{0}+1}, \ldots$, satisfies equation (14), it is denoted by $\left\{\bar{y}_{m}\right\}$, in this paper, and is called a solution of (14). It is easily known that equation (14) has a unique solution when given $k$ initial values $\bar{y}_{q_{0}}, \bar{y}_{q_{0}+1}, \ldots, \bar{y}_{q_{0}+k-1}$. If every $b_{m}$ is zero, equation (14) is a so-called homogeneous equation:

$$
\sum_{j=0}^{k} a_{j} \bar{y}_{m+j}=0, \quad m=q_{0}, q_{0}+1, \ldots
$$

A system of $k$ linearly independent solutions $\left\{\bar{y}_{m}^{(1)}\right\},\left\{\bar{y}_{m}^{(2)}\right\}, \ldots,\left\{\bar{y}_{m}^{(k)}\right\}$ of equation (15) is called a fundamental system of (15), and the general solution $\left\{\bar{y}_{m}\right\}$ of equation (15) can be written by 


$$
\bar{y}_{m}=\sum_{i=1}^{k} d_{i} \bar{y}_{m}^{(i)}, \quad m=q_{0}, q_{0}+1, \ldots
$$

where each $d_{i}$ is an arbitrary complex constant.

Call the algebraic equation

$$
\sum_{j=0}^{k} a_{j} \xi^{j}=0
$$

which corresponds to (14) or (15) the characteristic equation.

Proposition 1 (see $[35,38])$. Suppose $\xi_{1}, \xi_{2}, \ldots, \xi_{k_{0}}\left(k_{0} \leq k\right)$ are all different roots of the characteristic equation (17), and let $r_{j}$ denote the multiple number of $\xi_{j}$. Then, when $a_{0} \neq 0$, the $k=\sum_{j=1}^{k_{0}} r_{j}$ sequences with the elements $\bar{y}_{m}$ which can be equal to

$$
\xi_{j}^{m-q_{0}},\left(m-q_{0}\right) \xi_{j}^{m-q_{0}}, \ldots,\left(m-q_{0}\right)\left(m-q_{0}-1\right) \ldots\left(m-q_{0}-r_{j}+2\right) \xi_{j}^{m-q_{0}}, \quad j=1,2, \ldots, k_{0}
$$

form a fundamental system of equation (15), and when $a_{0}=0$, which means equation (17) has zero root and we further suppose $\xi_{1}=0$ is a $r_{1}$-tuple root of the characteristic equation (17), the $k=\sum_{j=1}^{k_{0}} r_{j}$ sequences with the elements $\bar{y}_{m}$ which can be equal to

$$
\begin{gathered}
\delta_{\left(m-q_{0}\right) q}, \quad q=0,1, \ldots, r_{1}-1, \\
\xi_{j}^{m-q_{0}},\left(m-q_{0}\right) \xi_{j}^{m-q_{0}}, \ldots,\left(m-q_{0}\right)\left(m-q_{0}-1\right) \ldots\left(m-q_{0}-r_{j}+2\right) \xi_{j}^{m-q_{0}}, \quad j=2,3, \ldots, k_{0},
\end{gathered}
$$

form a fundamental system of equation (15), where $\delta_{i j}$ is the Kronecker function:

$$
\delta_{i j}= \begin{cases}0, & \text { when } i \neq j, \\ 1, & \text { when } i=j .\end{cases}
$$

Remark 1. Some explanations about the proof of Proposition 1 are as follows:

(i) When $a_{0} \neq 0$ and $q_{0}=0, \mathrm{P}$. Henrici has given the proof in the famous book [38].

(ii) When $a_{0} \neq 0$ and $q_{0} \neq 0$, from the conclusion in the case that $a_{0} \neq 0$ and $q_{0}=0$, the proof is easily completed.

(iii) When $a_{0}=0$ and $q_{0}=0$, the corresponding part of Proposition 1 is based on the proof [38] in the case that $a_{0} \neq 0$ and $q_{0}=0$ mentioned in the above (i) and some conclusions in the case that $a_{0}=0$ and $q_{0}=0$ in [35], and we offer the explanations about the proof as follows. Firstly, $\xi_{1}=0$ is a $r_{1}$-tuple root of the characteristic equation (17) which means that $a_{0}=a_{1}=\cdots=a_{r_{1}-1}=0$, and it is easily further known as the $r_{1}$ sequences with the elements $\bar{y}_{m}=$ $\delta_{\left(m-q_{0}\right) q}\left(q=0,1, \ldots, r_{1}-1\right)$ are the solutions of equation (15). Secondly, based on the proof presented by P. Henrici mentioned in the above (i), we can know the sequences with the elements shown in (20) are solutions of equation (15) and that the solutions with the elements shown in (19) and (20) are linearly independent.

(iv) When $a_{0}=0$ and $q_{0} \neq 0$, from the conclusion in the case that $a_{0}=0$ and $q_{0}=0$, the proof is easily completed.

Proposition 2 (see [35]). When the initial values $\bar{y}_{q_{0}}=\bar{y}_{q_{0}+1}=\cdots=\bar{y}_{q_{0}+k-1}=0$, the solution $\left\{\bar{y}_{m}\right\}$ of equation (14) is given by

$$
\bar{y}_{m}=\sum_{s=q_{0}}^{m-k} b_{s} y_{m-s-1}^{*}, \quad m=q_{0}, q_{0}+1, \ldots
$$

where each $y_{m}^{*}$ satisfies the homogeneous equation:

$$
\left\{\sum_{j=0}^{k} a_{j} y_{m+j}^{*}=0, \quad m=0,1, \ldots, y_{0}^{*}=y_{1}^{*}=\cdots=y_{k-2}^{*}=0, \quad y_{k-1}^{*}=\frac{1}{a_{k}} .\right.
$$

Remark 2. In this paper, when $q<p$, we define $\sum_{i=p}^{q} Q_{i} \equiv 0$ and $\max _{p \leq i \leq q} Q_{i} \equiv 0$, where each $Q_{i}$ is any a given expression.
Remark 3. By Theorem 5.2 in [38], Proposition 2 is easily shown. 
Proposition 3 (see [35]). Suppose $\left\{\bar{y}_{m}^{(i)}\right\}(i=1,2, \ldots, k)$ is a fundamental system of equation (15) and $\left\{y_{m}^{*}\right\}$ is the solution of the homogeneous equation (23). Then, the general solution $\left\{\bar{y}_{m}\right\}$ of equation (14) can be expressed by

$$
\bar{y}_{m}=\sum_{i=1}^{k} c_{i} \bar{y}_{m}^{(i)}+\sum_{s=q_{0}}^{m-k} b_{s} y_{m-s-1}^{*}, \quad m=q_{0}, q_{0}+1, \ldots,
$$

where each $c_{i}$ is an arbitrary constant.

Proposition 4 (discrete Bellman inequality). Let $\mu_{1}, \mu_{2} \geq 0, h>0, \eta_{q_{0}}, \eta_{q_{0}+1}, \ldots, \eta_{N}$ be a sequence of nonnegative real numbers which satisfy

$$
\eta_{m} \leq \mu_{2}+\mu_{1} h \sum_{j=q_{0}}^{m-1} \eta_{j}, \quad m=q_{0}, q_{0}+1, \ldots, N .
$$

Then,

$$
\eta_{m} \leq \mu_{2} e^{\mu_{1}\left(m-q_{0}\right) h}, \quad m=q_{0}, q_{0}+1, \ldots, N .
$$

Definition 1. We say method (6) is zero-stable if the sequence $y_{\breve{k}}, y_{\breve{k}+1}, \ldots, y_{N}$ determined by method (6) applied to equation (1) $\in \mathbb{D}\left(L_{1}, L_{2}\right)$ with starting data $\psi \in \mathbf{C}_{m}[-\tau, 0], y_{0}:=\psi(0), y_{1}, y_{2}, \ldots, y_{\bar{k}-1} \in \mathbf{R}^{m} \quad$ (the method (6) starts with $n=k-k)$ and the sequence $z_{\breve{k}}, z_{\breve{k}+1}, \ldots, z_{N}$ determined by the perturbation equations

$$
\left\{\begin{array}{l}
z_{n i}^{h}(t)=\prod_{n i}^{h}\left(t ; \chi, z_{1}, z_{2}, \ldots, z_{n+k}\right)+\gamma_{n i}(t), \quad-\tau \leq t \leq t_{n+i}, i=0,1, \ldots, k, \\
\sum_{i=0}^{k} \alpha_{i} z_{n+i}=h\left(\left(\sum_{i=0}^{k} \beta_{i} f\left(t_{n+i}, z_{n+i}, z_{n i}^{h}(\cdot)\right)\right)+\varepsilon_{n+k}\right), \quad n=\breve{k}-k, \breve{k}-k+1, \ldots, N-k,
\end{array}\right.
$$

with starting data $\chi \in \mathbf{C}_{m}[-\tau, 0], z_{0}:=\chi(0), z_{1}, z_{2}, \ldots, z_{\breve{k-1}} \in \mathbf{R}^{m}$, satisfy

$$
\max _{\breve{k} \leq n \leq N}\left\|z_{n}-y_{n}\right\| \leq C\left(\max _{1 \leq j \leq k-1}\left\|z_{j}-y_{j}\right\|+\max _{t \in[-\tau, 0]}\|\chi(t)-\psi(t)\|+\max _{\breve{k}-k \leq s \leq N-k} \max _{0 \leq i \leq k} \max _{t \in\left[-\tau, t_{s+i}\right]}\left\|\gamma_{s i}(t)\right\|+\max _{\bar{k} \leq j \leq N}\left\|\varepsilon_{j}\right\|\right)
$$

where $\gamma_{n i}(t) \in \mathbf{C}_{m}\left[-\tau, t_{n+i}\right]$ and $\varepsilon_{n+k} \in \mathbf{R}^{m}$ are both perturbations, $0<h \leq h_{0}$, the constant $h_{0}>0$ depends on method (6), $L_{1}$ and $L_{2}$, and the constant $C$ is independent of $h$.

Definition 2. We say method (6) satisfies the root condition if for all roots of the characteristic polynomial $\rho(\xi)=\sum_{i=0}^{k} \alpha_{i} \xi^{i}$, the moduli of them are all no larger than 1 , and among them any root whose modulus equals 1 is simple root.

Theorem 1. Method (6) is zero-stable if and only if it satisfies the root condition.

Proof. Firstly, we prove method (6) satisfies the root condition if it is zero-stable. Consider the ODEs:

$$
\begin{cases}y^{\prime}(t)=f(t, y(t)), & t \in[0, T], \\ y(t)=\eta, & t=0,\end{cases}
$$

which are a special case of $(1)$, where $T \in(0,+\infty), \eta \in \mathbf{R}^{m}$, and the continuous mapping $f:[0, T] \times \mathbf{R}^{m} \longrightarrow \mathbf{R}^{m}$ satisfies the classical Lipschitz condition:

$$
\left\|f\left(t, u_{1}\right)-f\left(t, u_{2}\right)\right\| \leq L_{1}\left\|u_{1}-u_{2}\right\|, \quad \forall t \in[0, T], u_{1}, u_{2} \in \mathbf{R}^{m},
$$

where $L_{1}$ is a classical Lipschitz constant. For the ODEs (29), method (6) degrades into its mother method (5). Method (6) is zero-stable implies that its mother method (5) is zerostable, which means method (6) should satisfy the root condition if it is zero-stable [35, 40].

We assume that method (6) satisfies the root condition, and now we prove it is zero-stable.

Denote $\delta_{n}=z_{n}-y_{n}, n=0,1, \ldots, N$. From (6) and (27), we obtain

$$
\sum_{i=0}^{k} \alpha_{i} \delta_{n+i}=\bar{b}_{n}, \quad n=\breve{k}-k, \breve{k}-k+1, \ldots, N-k,
$$

where 


$$
\bar{b}_{n}=h\left(\left(\sum_{i=0}^{k} \beta_{i}\left(f\left(t_{n+i}, z_{n+i}, z_{n i}^{h}(\cdot)\right)-f\left(t_{n+i}, y_{n+i}, y_{n i}^{h}(\cdot)\right)\right)\right)+\varepsilon_{n+k}\right) .
$$

Using Proposition 1 and considering that all $\alpha_{i}$ are real constants, we can find a real fundamental system $[38]\left\{\omega_{n}^{(i)}\right\}(i=1,2, \ldots, k)$ of the following scalar homogeneous equation:

$$
\sum_{j=0}^{k} \alpha_{j} \omega_{n+j}=0, \quad n=\breve{k}-k, \breve{k}-k+1, \ldots, N-k
$$

$$
\delta_{n}=\sum_{i=1}^{k} c_{i} \omega_{n}^{(i)}+\sum_{s=\bar{k}-k}^{n-k} \bar{b}_{s} y_{n-s-1}^{*}, n=\breve{k}-k, \breve{k}-k+1, \ldots, N \text {, }
$$

where each $c_{i} \in \mathbf{R}^{m}$ is a constant vector and each $y_{n}^{*}$ satisfies the scalar homogeneous equation:

and by applying Proposition 3 to (31), we obtain

$$
\left\{\sum_{j=0}^{k} \alpha_{j} y_{n+j}^{*}=0, \quad n=0,1, \ldots, y_{0}^{*}=y_{1}^{*}=\cdots=y_{k-2}^{*}=0, \quad y_{k-1}^{*}=\frac{1}{\alpha_{k}} .\right.
$$

From the assumption that method (6) satisfies the root condition, we can conclude for all $\omega_{n}^{(i)}$ and $y_{n-s-1}^{*}$ in (34), there exists a constant $M$ which is only dependent on the mother method (5) such that

$$
\left|\omega_{n}^{(i)}\right| \leq M,\left|y_{n-s-1}^{*}\right| \leq M \text {. }
$$

It follows from (34) and (36) that

$$
\left\|\delta_{n}\right\| \leq M\left(\sum_{i=1}^{k}\left\|c_{i}\right\|+\sum_{s=\bar{k}-k}^{n-k}\left\|\bar{b}_{s}\right\|\right), \quad n=\breve{k}-k, \breve{k}-k+1, \ldots, N .
$$

Setting $n=\breve{k}-k, \breve{k}-k+1, \ldots, \breve{k}-1$ in (34), we thus find

$$
c_{i}=\sum_{j=1}^{k}\left(W^{-1}\right)_{i j} \delta_{\breve{k-k-1+j}}, \quad i=1,2, \ldots, k,
$$

where the matrix

$$
W=\left[\begin{array}{cccc}
\omega_{\vec{k}-k}^{(1)} & \omega_{\vec{k}-k}^{(2)} & \cdots & \omega_{\breve{k}-k}^{(k)} \\
\omega_{\vec{k}-k+1}^{(1)} & \omega_{\vec{k}-k+1}^{(2)} & \cdots & \omega_{\vec{k}-k+1}^{(k)} \\
\cdots & \cdots & \cdots & \cdots \\
\omega_{\vec{k}-1}^{(1)} & \omega_{\breve{k}-1}^{(2)} & \cdots & \omega_{\breve{k}-1}^{(k)}
\end{array}\right] .
$$

By (38), we know

$$
\sum_{i=1}^{k}\left\|c_{i}\right\| \leq\left\|\left|W^{-1}\right|\right\| \max _{\breve{k}-k \leq j \leq \breve{k}-1}\left\|\delta_{j}\right\|
$$

where $\left\|\left|W^{-1}\right|\right\|=\sum_{i, j=1}^{k}\left|\left(W^{-1}\right)_{i j}\right|$. Combining (37) with (40), we have

$$
\left\|\delta_{n}\right\| \leq M\left\|\left|W^{-1}\right|\right\| \max _{\breve{k}-k \leq j \leq \bar{k}-1}\left\|\delta_{j}\right\|+M \sum_{s=\breve{k}-k}^{n-k}\left\|\bar{b}_{s}\right\|, \quad n=\breve{k}-k, \breve{k}-k+1, \ldots, N .
$$

It is known from (2), (6), (11), (27), and (32) that 


$$
\begin{aligned}
& \left\|\bar{b}_{s}\right\| \leq h \sum_{i=0}^{k}\left(\left|\beta_{i}\right|\left(\left\|f\left(t_{s+i}, z_{s+i}, z_{s i}^{h}(\cdot)\right)-f\left(t_{s+i}, y_{s+i}, y_{s i}^{h}(\cdot)\right)\right\|\right)\right)+h\left\|\varepsilon_{s+k}\right\| \\
& \leq h \sum_{i=0}^{k}\left(| \beta _ { i } | \left(\| f\left(t_{s+i}, z_{s+i}, z_{s i}^{h}(\cdot)\right)-f\left(t_{s+i}, y_{s+i}, z_{s i}^{h}(\cdot)\right)\right.\right. \\
& \left.\left.+f\left(t_{s+i}, y_{s+i}, z_{s i}^{h}(\cdot)\right)-f\left(t_{s+i}, y_{s+i}, y_{s i}^{h}(\cdot)\right) \|\right)\right)+h\left\|\varepsilon_{s+k}\right\| \\
& \leq h \sum_{i=0}^{k}\left(| \beta _ { i } | \left(\left\|f\left(t_{s+i}, z_{s+i}, z_{s i}^{h}(\cdot)\right)-f\left(t_{s+i}, y_{s+i}, z_{s i}^{h}(\cdot)\right)\right\|\right.\right. \\
& \left.\left.+\left\|f\left(t_{s+i}, y_{s+i}, z_{s i}^{h}(\cdot)\right)-f\left(t_{s+i}, y_{s+i}, y_{s i}^{h}(\cdot)\right)\right\|\right)\right)+h\left\|\varepsilon_{s+k}\right\| \\
& \leq h \sum_{i=0}^{k}\left(\left|\beta_{i}\right|\left(L_{1}\left\|\delta_{s+i}\right\|+L_{2} \max _{t \in\left[-\tau, t_{s+i}\right]}\left\|z_{s i}^{h}(t)-y_{s i}^{h}(t)\right\|\right)\right)+h\left\|\varepsilon_{s+k}\right\| \\
& \leq h \sum_{i=0}^{k}\left(| \beta _ { i } | \left(L_{1}\left\|\delta_{s+i}\right\|+L_{2} \max _{t \in\left[-\tau, t_{s+i}\right]} \| \prod_{s i}^{h}\left(t ; \chi, z_{1}, z_{2}, \ldots, z_{s+k}\right)\right.\right. \\
& \left.\left.-\prod_{s i}^{h}\left(t ; \psi, y_{1}, y_{2}, \ldots, y_{s+k}\right)\left\|+L_{2} \max _{t \in\left[-\tau, t_{s+i}\right]}\right\| \gamma_{s i}(t) \|\right)\right)+h\left\|\varepsilon_{s+k}\right\| \\
& \leq h \sum_{i=0}^{k}\left(| \beta _ { i } | \left(L_{1}\left\|\delta_{s+i}\right\|+L_{2} c_{\pi} \max \left\{\max _{1 \leq \bar{i} \leq s+k}\left\|\delta_{i}\right\|, \max _{t \in[-\tau, 0]}\|\chi(t)-\psi(t)\|\right\}\right.\right. \\
& \left.\left.+L_{2} \max _{t \in\left[-\tau, t_{s+i}\right]}\left\|\gamma_{s i}(t)\right\|\right)\right)+h\left\|\varepsilon_{s+k}\right\|, \quad s=\breve{k}-k, \breve{k}-k+1, \ldots, N-k .
\end{aligned}
$$

Then, (42) yields

$$
\begin{aligned}
\sum_{s=\breve{k}-k}^{n-k}\left\|\bar{b}_{s}\right\| \leq & h \sum_{i=0}^{k} \sum_{s=\breve{k}-k}^{n-k}\left(\left|\beta_{i}\right|\left(L_{1}\left\|\delta_{s+i}\right\|+L_{2} c_{\pi} \max \left\{\max _{1 \leq \bar{i} \leq s+k}\left\|\delta_{i}\right\|, \max _{t \in[-\tau, 0]}\|\chi(t)-\psi(t)\|\right\}+L_{2} \max _{t \in\left[-\tau, t_{s+i}\right]}\left\|\gamma_{s i}(t)\right\|\right)\right)+h \sum_{s=\bar{k}-k}^{n-k}\left\|\varepsilon_{s+k}\right\| \\
\leq & h \sum_{i=0}^{k} \sum_{j=\breve{k}-k}^{n}\left(\left|\beta_{i}\right| L_{1}\left\|\delta_{j}\right\|\right)+L_{2} c_{\pi} h \sum_{i=0}^{k} \sum_{s=\breve{k}-k}^{n-k}\left(\left|\beta_{i}\right| \max \left\{\max _{1 \leq \bar{i} \leq s+k}\left\|\delta_{\bar{i}}\right\|, \max _{t \in[-\tau, 0]}\|\chi(t)-\psi(t)\|\right\}\right) \\
& +h L_{2} \sum_{i=0}^{k} \sum_{s=\bar{k}-k}^{n-k}\left(\left|\beta_{i}\right| \max _{t \in\left[-\tau, t_{s+i}\right]}\left\|\gamma_{s i}(t)\right\|\right)+h \sum_{s=\breve{k}-k}^{n-k}\left\|\epsilon_{s+k}\right\|, \quad n=\breve{k}, \breve{k}+1, \ldots, N .
\end{aligned}
$$

Setting $\beta=\max _{0 \leq i \leq k}\left|\beta_{i}\right|$ and noticing (43), we have

$$
\begin{aligned}
& \sum_{s=\breve{k}-k}^{n-k}\left\|\bar{b}_{s}\right\| \leq(k+1) h \beta L_{1} \sum_{j=\breve{k}-k}^{n}\left\|\delta_{j}\right\|+(k+1) h \beta L_{2} c_{\pi} \sum_{s=\breve{k}-k}^{n-k} \max \left\{\max _{1 \leq \bar{i} \leq s+k}\left\|\delta_{\bar{i}}\right\|, \max _{t \in[-\tau, 0]}\|\chi(t)-\psi(t)\|\right\}+ \\
& (k+1) h \beta L_{2} \sum_{s=\breve{k}-k}^{n-k} \max _{0 \leq i \leq k} \max _{t \in\left[-\tau, t_{s+i}\right]}\left\|\gamma_{s i}(t)\right\|+t_{n} \max _{\breve{k} \leq j \leq n}\left\|\varepsilon_{j}\right\|, \quad n=\breve{k}, \breve{k}+1, \ldots, N .
\end{aligned}
$$

Denote

$$
Q_{0}=\left\|\delta_{0}\right\|, Q_{n}=\max \left\{\left\|\delta_{n}\right\|, \max _{0 \leq i \leq n-1}\left\|\delta_{i}\right\|\right\} \leq\left\|\delta_{n}\right\|+Q_{n-1}, \quad n \geq 1
$$


Combining (44) with (45), we conclude

$$
\begin{aligned}
& \sum_{s=\breve{k}-k}^{n-k}\left\|\bar{b}_{s}\right\| \leq(k+1) h \beta L_{1} \sum_{j=\breve{k}-k}^{n} Q_{j}+(k+1) h \beta L_{2} c_{\pi} \sum_{j=\breve{k}}^{n} Q_{j}+(k \\
& \quad+1) h \beta L_{2} c_{\pi} \sum_{s=\breve{k}-k}^{n-k} \max _{t \in[-\tau, 0]}\|\chi(t)-\psi(t)\|+(k \\
& \quad+1) h \beta L_{2} \sum_{s=\breve{k}-k}^{n-k} \max _{0 \leq i \leq k} \max _{t \in\left[-\tau, t_{s+i}\right]}\left\|\gamma_{s i}(t)\right\|+t_{n} \max _{\breve{k} \leq j \leq n}\left\|\varepsilon_{j}\right\|, \\
& n=\breve{k}, \breve{k}+1, \ldots, N .
\end{aligned}
$$

$$
\begin{aligned}
& \left\|\delta_{n}\right\| \leq M\left\|W^{-1} \mid\right\| \max _{\bar{k}-k \leq j \leq \bar{k}-1}\left\|\delta_{j}\right\|+(k+1) h \beta M\left(L_{1}+L_{2} c_{\pi}\right) \sum_{j=k-k}^{n} Q_{j}+(k+1) \beta L_{2} c_{\pi} M t_{n} \max _{t \in[-\tau, 0]}\|\chi(t)-\psi(t)\| \\
& +(k+1) \beta L_{2} M t_{n} \max _{\bar{k}-k \leq s \leq n-k} \max _{0 \leq i \leq k} \max _{t \in\left[-\tau, t_{s+i}\right]}\left\|\gamma_{s i}(t)\right\|+M t_{n} \max _{\substack{k \leq j \leq n}}\left\|\mathcal{E}_{j}\right\|, \quad n=\breve{k}-k, \breve{k}-k+1, \ldots, N .
\end{aligned}
$$

By (45), we have

$$
\sum_{j=\breve{k}-k}^{n} Q_{j}=\sum_{j=\breve{k}-k}^{n-1} Q_{j}+Q_{n} \leq 2 \sum_{j=\breve{k}-k}^{n-1} Q_{j}+\left\|\delta_{n}\right\|, \quad n=\breve{k}, \breve{k}+1, \ldots, N .
$$

It can be concluded from (47) and (48) that

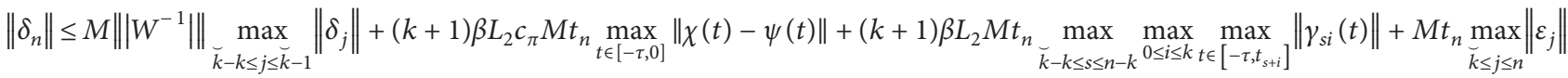

$$
\begin{aligned}
& +(k+1) h \beta M\left(L_{1}+L_{2} c_{\pi}\right)\left\|\delta_{n}\right\|+2(k+1) h \beta M\left(L_{1}+L_{2} c_{\pi}\right) \sum_{j=\breve{k}-k}^{n-1} Q_{j}, \quad n=\breve{k}, \breve{k}+1, \ldots, N .
\end{aligned}
$$

Let

$$
\begin{aligned}
& \tilde{\alpha}_{n}=M\left\|\left|W^{-1}\right|\right\| \max _{\breve{k}-k \leq j \leq k-1}\left\|\delta_{j}\right\|+(k+1) \beta L_{2} c_{\pi} M t_{n} \max _{t \in[-\tau, 0]}\|\chi(t)-\psi(t)\|+(k+1) \beta L_{2} M t_{n} \max _{k-k \leq s \leq n-k} \max _{0 \leq i \leq k} \max _{t \in\left[-\tau, t_{s+i}\right]}\left\|\gamma_{s i}(t)\right\| \\
& +M t t_{n} \max _{k \leq j \leq n}\left\|\varepsilon_{j}\right\| \\
& +\max _{t \in[-\tau, 0]}\|\chi(t)-\psi(t)\|+\max _{1 \leq j \leq k-1}\left\|\delta_{j}\right\|, \quad n=0,1, \ldots, N
\end{aligned}
$$


From (49) and (50), we know that, for $n=0,1, \ldots, N$,

$$
\begin{gathered}
\left\|\delta_{n}\right\| \leq \widetilde{\alpha}_{n}+(k+1) h \beta M\left(L_{1}+L_{2} c_{\pi}\right)\left\|\delta_{n}\right\|+2(k+1) h \beta M\left(L_{1}+L_{2} c_{\pi}\right) \sum_{j=\bar{k}-k}^{n-1} Q_{j}, \\
\left(1-(k+1) h \beta M\left(L_{1}+L_{2} c_{\pi}\right)\right)\left\|\delta_{n}\right\| \leq \widetilde{\alpha}_{n}+2(k+1) h \beta M\left(L_{1}+L_{2} c_{\pi}\right) \sum_{j=\breve{k}-k}^{n-1} Q_{j} .
\end{gathered}
$$

For any given $v_{1} \in(0,1)$, set

$$
h_{0}=\min \left\{\frac{1-v_{1}}{(k+1) \beta M\left(L_{1}+L_{2} c_{\pi}\right)}, h_{\varphi}\right\} \text {, }
$$

which depends on method (6), $L_{1}$ and $L_{2}$, where $h_{\varphi}$ is defined in Section 2. Noticing $k-k \geq 0$, when $0<h \leq h_{0}$, from (52), we obtain

$$
\begin{array}{r}
\left\|\delta_{n}\right\| \leq v_{1}^{-1} \widetilde{\alpha}_{n}+2 v_{1}^{-1}(k+1) h \beta M\left(L_{1}+L_{2} c_{\pi}\right) \sum_{j=\bar{k}-k}^{n-1} Q_{j} \\
\leq v_{1}^{-1} \widetilde{\alpha}_{n}+2 v_{1}^{-1}(k+1) h \beta M\left(L_{1}+L_{2} c_{\pi}\right) \sum_{j=0}^{n-1} Q_{j}, \quad n=0,1, \ldots, N .
\end{array}
$$

Because $\widetilde{\alpha}_{n}$ does not decrease as $n$ increases, when $n \geq 1$ and $0 \leq i \leq n-1$, from (54), we know

It is concluded from (54) and (55) that

$$
\left\|\delta_{i}\right\| \leq v_{1}^{-1} \widetilde{\alpha}_{n}+2 v_{1}^{-1}(k+1) h \beta M\left(L_{1}+L_{2} c_{\pi}\right) \sum_{j=0}^{n-1} Q_{j}, \quad 0<h \leq h_{0} .
$$

$$
Q_{n} \leq v_{1}^{-1} \widetilde{\alpha}_{n}+2 v_{1}^{-1}(k+1) h \beta M\left(L_{1}+L_{2} c_{\pi}\right) \sum_{j=0}^{n-1} Q_{j}, \quad 0<h \leq h_{0}, n=0,1, \ldots, N
$$

which implies

$$
Q_{i} \leq v_{1}^{-1} \widetilde{\alpha}_{n}+2 v_{1}^{-1}(k+1) h \beta M\left(L_{1}+L_{2} c_{\pi}\right) \sum_{j=0}^{i-1} Q_{j}, \quad 0<h \leq h_{0}, i=0,1, \ldots, n, n=0,1, \ldots, N
$$

From (57) and Proposition 4, we have

$$
Q_{n} \leq v_{1}^{-1} \widetilde{\alpha}_{n} e^{2 v_{1}^{-1}(k+1) h n \beta M\left(L_{1}+L_{2} c_{n}\right)}, \quad 0<h \leq h_{0}, n=0,1, \ldots, N
$$




$$
\begin{aligned}
& \delta_{n} \leq Q_{n} \leq v_{1}^{-1} \widetilde{\alpha}_{n} e^{2 v_{1}^{-1}(k+1) t_{n} \beta M\left(L_{1}+L_{2} c_{\pi}\right)}, \quad 0<h \leq h_{0}, n=0,1, \ldots, N, \\
& \max _{0 \leq i \leq N}\left\|\delta_{i}\right\| \leq v_{1}^{-1} \widetilde{\alpha}_{N} e^{2 v_{1}^{-1}(k+1) T \beta M\left(L_{1}+L_{2} c_{\pi}\right)} \leq v_{1}^{-1} e^{2 v_{1}^{-1}(k+1) T \beta M\left(L_{1}+L_{2} c_{\pi}\right)}\left(\left(M\left\|\left|W^{-1}\right|\right\|+1\right) \max _{1 \leq j \leq k-1}\left\|\delta_{j}\right\|\right. \\
& +\left((k+1) \beta L_{2} c_{\pi} M T+M\left\|\left|W^{-1}\right|\right\|+1\right) \max _{t \in[-\tau, 0]}\|\chi(t)-\psi(t)\| \\
& \left.+(k+1) \beta L_{2} M T \max _{\breve{k}-k \leq s \leq N-k} \max _{0 \leq i \leq k} \max _{t \in\left[-\tau, t_{s+i}\right]}\left\|\gamma_{s i}(t)\right\|+M T \max _{\breve{k}_{\leq} \leq N}\left\|\mathcal{E}_{j}\right\|\right) \\
& \leq C\left(\max _{1 \leq j \leq k-1}\left\|\delta_{j}\right\|+\max _{t \in[-\tau, 0]}\|\chi(t)-\psi(t)\|+\max _{\breve{k}-k \leq s \leq N-k} \max _{0 \leq i \leq k} \max _{t \in\left[-\tau, t_{s+i}\right]}\left\|\gamma_{s i}(t)\right\|+\max _{\mathcal{k}^{\prime} \leq j \leq N}\left\|\varepsilon_{j}\right\|\right), \quad 0<h \leq h_{0},
\end{aligned}
$$

where

$$
C=v_{1}^{-1} e^{2 v_{1}^{-1}(k+1) T \beta M\left(L_{1}+L_{2} c_{\pi}\right)} \max \left\{M\left\|\left|W^{-1}\right|\right\|+1,(k+1) \beta L_{2} c_{\pi} M T+M\left\|\left|W^{-1}\right|\right\|+1,(k+1) \beta L_{2} M T, M T\right\},
$$

which is independent of $h$. This completes the proof.

\section{Consistency and Convergence Analyses}

In this section, for equation (1), we assume the mapping $f$ possesses sufficiently high-order continuous partial derivatives, and its true solution $y(t)$ possesses sufficiently highorder continuous derivatives on the interval $[0, T]$ and the constants $M_{i}$ used later which are defined as

$$
M_{i}=\sup _{0 \leq t \leq T}\left\|\frac{\mathrm{d}^{i} y(t) \|}{\mathrm{d} t^{i}}\right\|
$$

are all of moderate size except for some special cases such as in the transient phase of a stiff problem.

Definition 3. It is said the piecewise Lagrangian interpolation operator $\Pi_{n i}^{h}$ in (6) is consistent of order $p(p \geq 1)$ if, for any given function $u$ : $[-\tau, T] \longrightarrow \mathbf{R}^{m}$ which is sufficiently differentiable on the subinterval $[0, T]$, there exists

$$
\max _{-\tau \leq t \leq t_{n+i}}\left\|u(t)-\prod_{n i}^{h}\left(t ; \phi, u\left(t_{1}\right), u\left(t_{2}\right), \ldots, u\left(t_{n+k}\right)\right)\right\| \leq \hat{g} h^{p}
$$

where the function $\phi$ is a restriction of the function $u(t)$ on the subinterval $[-\tau, 0]$ and the constant $\hat{g}$ only depends on some $\tilde{M}_{j}$ defined as $\widetilde{M}_{j}=\sup _{t \in[0, T]}\left(\left\|\mathrm{d}^{j} u(t) / \mathrm{d} t^{j}\right\|\right)$.

Remark 4. By Definition 3 and the Lagrangian interpolation theorem, we know the piecewise interpolation operators $\Pi_{n i}^{h}$ in (6) which based on $p(p \geq 0)$-degree Lagrangian interpolation polynomial are all consistent of order $p+1$.

Definition 4. It is said method (6) is consistent of order $p$ if its mother method (5) and the piecewise interpolation operators

$\Pi_{n i}^{h}(n=k-k, \breve{k}-k+1, \ldots, N-k, i=0,1, \ldots, k)$ are all consistent of order $p$.

Remark 5. As for the consistency of the mother method (5), please see references $[35,38,40]$.

Definition 5. Method (6) is said to be convergent of order $p$ if, for the sequence $\left\{y_{\breve{k}}, y_{\breve{k}_{+1}}, \ldots, y_{N}\right\}$ determined by method (6) applied to any given equations (1) in $\mathbb{D}\left(L_{1}, L_{2}\right)$ with the starting data $\psi \in \mathbf{C}_{m}[-\tau, 0], y_{0}:=\psi(0), y_{1}, y_{2}, \ldots, y_{\breve{k}-1} \in \mathbf{R}^{m} \quad$ (the method (6) starts with $n=k-k$ ), there exists a sufficiently small positive number $\widetilde{h}$, such that

$$
\left\|y\left(t_{n}\right)-y_{n}\right\| \leq C_{0}\left(t_{n}\right) \max \left\{\max _{-\tau \leq t \leq 0}\|\varphi(t)-\psi(t)\|, \max _{1 \leq j \leq \bar{k}-1}\left\|y\left(t_{j}\right)-y_{j}\right\|\right\}+C_{1}\left(t_{n}\right) h^{p}, \quad n=\breve{k}, \breve{k}+1, \ldots, N, 0<h \leq \widetilde{h}
$$

where the continuous functions $C_{0}(t)$ and $C_{1}(t)$ depend on $t$, method (6), $L_{1}, L_{2}$, and some $M_{i}$.

Theorem 2. If method (6) is consistent of order $p$ and satisfies the root condition, it is convergent of order $p$.
Proof. Assume method (6) is consistent of order $p$ and satisfies the root condition. In this proving course, we always assume $0<h \leq h_{0}$, where $h_{0}$ is defined by (53). Using method (6) to solve any given equations $(1) \in \mathbb{D}\left(L_{1}, L_{2}\right)$, we easily know the true solution $y(t)$ obviously satisfies the perturbation equations: 


$$
\left\{\begin{array}{l}
y_{n i}(t)=\prod_{n i}^{h}\left(t ; \varphi, y\left(t_{1}\right), y\left(t_{2}\right), \ldots, y\left(t_{n+k}\right)\right)+\gamma_{n i}(t), \quad-\tau \leq t \leq t_{n+i}, i=0,1, \ldots, k, \\
\sum_{i=0}^{k} \alpha_{i} y\left(t_{n+i}\right)=h\left(\left(\sum_{i=0}^{k} \beta_{i} f\left(t_{n+i}, y\left(t_{n+i}\right), y_{n i}(\cdot)\right)\right)+\frac{1}{h} T_{n+k}\right), \quad n=\breve{k}-k, \breve{k}-k+1, \ldots, N-k,
\end{array}\right.
$$

where

$$
\begin{aligned}
\gamma_{n i}(t) & =y(t)-\prod_{n i}^{h}\left(t ; \varphi, y\left(t_{1}\right), y\left(t_{2}\right), \ldots, y\left(t_{n+k}\right)\right), \quad-\tau \leq t \leq t_{n+i}, i=0,1, \ldots, k \\
T_{n+k} & =\sum_{i=0}^{k}\left(\alpha_{i} y\left(t_{n+i}\right)-h \beta_{i} f\left(t_{n+i}, y\left(t_{n+i}\right), y_{n i}(\cdot)\right)\right) .
\end{aligned}
$$

From (1) and (67), we conclude

$$
T_{n+k}=\sum_{i=0}^{k}\left(\alpha_{i} y\left(t_{n+i}\right)-h \beta_{i} y^{\prime}\left(t_{n+i}\right)\right), \quad n=\breve{k}-k, \breve{k}-k+1, \ldots, N-k
$$

Since the mother method (5) is consistent of order $p$, equation (68) implies [35, 38, 40]

$$
\left\|T_{n+k}\right\| \leq \widetilde{C} h^{p+1}, \quad n=\breve{k}-k, \breve{k}-k+1, \ldots, N-k,
$$

where the constant $\widetilde{C}$ only depends on the mother method (5) and $M_{p+1}$ defined above. Because all the interpolation operators $\Pi_{n i}^{h}(n=k-k, \breve{k}-k+1, \ldots, N-k, i=0,1, \ldots, k)$ are consistent of order $p$, by Definition 3, we obtain

$$
\max _{\breve{k}-k \leq n \leq N-k} \max _{0 \leq i \leq k} \max _{-\tau \leq t \leq t_{n+i}}\left\|\gamma_{n i}(t)\right\| \leq \hat{g} h^{p}
$$

where the constant $\hat{g}$ only depends on some $M_{i}$ defined above. By Theorem 1 and assumption that method (6) satisfies the root condition, we know method (6) is zerostable, which tells us that inequality (59) holds. From (6), (27), (50), (59), (65), (69), and (70), we further conclude that

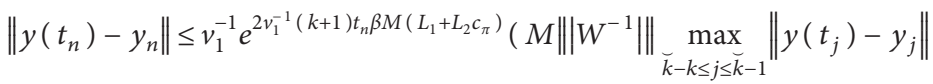

$$
\begin{aligned}
& +(k+1) \beta L_{2} c_{\pi} M t_{n} \max _{t \in[-\tau, 0]}\|\varphi(t)-\psi(t)\|+(k+1) \beta L_{2} M t_{n} \max _{\breve{k}-k \leq s \leq n-k} \max _{0 \leq i \leq k} \max _{t \in\left[-\tau, t_{s+i}\right]}\left\|\gamma_{s i}(t)\right\| \\
& \left.+\frac{M t_{n}}{h} \max _{\bar{k} \leq j \leq n}\left\|T_{j}\right\|+\max _{t \in[-\tau, 0]}\|\varphi(t)-\psi(t)\|+\max _{1 \leq j \leq \breve{k}-1}\left\|y\left(t_{j}\right)-y_{j}\right\|\right) \\
& \leq v_{1}^{-1} e^{2 v_{1}^{-1}(k+1) t_{n} \beta M\left(L_{1}+L_{2} c_{\pi}\right)}\left(\max _{t \in[-\tau, 0]}\|\varphi(t)-\psi(t)\|+\max _{1 \leq j \leq \bar{k}-1}\left\|y\left(t_{j}\right)-y_{j}\right\|+M\left\|W^{-1} \mid\right\|_{\breve{k}-k \leq j \leq \grave{k}-1} \max _{\|}\left\|y\left(t_{j}\right)-y_{j}\right\|\right. \\
& \left.+(k+1) \beta L_{2} c_{\pi} M t_{n} \max _{t \in[-\tau, 0]}\|\varphi(t)-\psi(t)\|+(k+1) \beta L_{2} M t_{n} \hat{g} h^{p}+M t_{n} \widetilde{C} h^{p}\right) \\
& \leq \widehat{C}_{0}\left(t_{n}\right) \max \left\{\max _{t \in[-\tau, 0]}\|\varphi(t)-\psi(t)\|, \max _{1 \leq j \leq \bar{k}-1}\left\|y\left(t_{j}\right)-y_{j}\right\|\right\}+\widehat{C}_{1}\left(t_{n}\right) h^{p}, \quad n=\breve{k}, \breve{k}+1, \ldots, N,
\end{aligned}
$$

where 


$$
\begin{array}{r}
\widehat{C}_{0}(t)=v_{1}^{-1}\left(M\left\|\left|W^{-1}\right|\right\|+(k+1) \beta L_{2} c_{\pi} M t+2\right) e^{2 v_{1}^{-1}(k+1) t \beta M\left(L_{1}+L_{2} c_{\pi}\right)}, \\
\widehat{C}_{1}(t)=v_{1}^{-1}\left((k+1) \beta L_{2} M \widehat{g}+M \widetilde{C}\right) t e^{2 v_{1}^{-1}(k+1) t \beta M\left(L_{1}+L_{2} c_{\pi}\right)},
\end{array}
$$

and $v_{1}, M,\left\|\left|W^{-1}\right|\right\|$, and $\beta$ defined in the course of proving Theorem 1 in Section 3. It can be seen that the continuous functions $\widehat{C}_{0}(t)$ and $\widehat{C}_{1}(t)$ depend on $t$, method (6), $L_{1}, L_{2}$, and some $M_{i}$. This completes the proof.

Remark 6. In this paper, the strict condition (2) can be generally weakened as the inequalities in (2) hold only in some neighborhood of the true solution $y(t)$ of the equations $[35,36,38,41]$.

\section{Numerical Examples}

In this section, the linear multistep methods formed as (6) are used to solve VFDEs formed as $(1) \in \mathbb{D}\left(L_{1}, L_{2}\right)$, and for convenience, we give the starting data $\psi(t), y_{0}, y_{1}, y_{2}, \ldots, y_{\bar{k}_{-1}}$ according to true solution, i.e., let

$$
\begin{aligned}
\psi(t) & =\varphi(t),-\tau \leq t \leq 0, \\
y_{0} & =\varphi(0), y_{i}=y\left(t_{i}\right), \quad i=1,2, \ldots, \breve{k}-1 .
\end{aligned}
$$

For a method formed as (6) whose stepsize is $h$, we denote the maximum global error:

$$
E(h)=\max _{0 \leq n \leq N} \max _{1 \leq i \leq m}\left|y_{i}\left(t_{n}\right)-y_{n i}\right|,
$$

where $y_{i}\left(t_{n}\right)$ and $y_{n i}$ are the $i$ th components of the true solution $y\left(t_{n}\right)$ and the approximation solution $y_{n}$, respectively, and furthermore, according to $[36,41]$, the observing order $\tilde{p}$ of the numerical method is defined as

$$
\widetilde{p}=\log _{2} \frac{E(h)}{E(h / 2)} .
$$

In order to adequately verify the correctness of the theory presented in this paper, we use various different zerostable mother methods including the Adams-Bashforth methods [38, 42], the Adams-Moulton method [38, 43], the Backward Differentiation Formula (BDF) method, and the Milne-Simpson method [44, 45] with corresponding piecewise Lagrangian interpolation operator to build the methods formed as (6), where each mother method and its corresponding interpolation operator are both consistent of the same order $p(2 \leq p \leq 4)$. According to Definition 4 , these methods are consistent of order $p$, and by Theorem 2, we know they are convergent of order $p$. For convenience, these methods are named the same as their corresponding mother methods. For simplicity, let $\mathrm{AB} p$ denote the Adams-Bashforth method which is consistent of order $p$, let AM $p$ denote the Adams-Moulton method which is consistent of order $p$, let $\mathrm{B} p$ denote the BDF method which is consistent of order $p$, and let MS4 denote the MilneSimpson method which is consistent of order 4 .

Remark 7. We list the mother methods used in this section as follows:

(i) The Adams-Bashforth method which is consistent of order 2 takes the form

$$
-y_{n+1}+y_{n+2}=h\left(-\frac{1}{2} f\left(t_{n}, y_{n}\right)+\frac{3}{2} f\left(t_{n+1}, y_{n+1}\right)\right) \text {. }
$$

(ii) The Adams-Bashforth method which is consistent of order 3 takes the form

$$
-y_{n+2}+y_{n+3}=h\left(\frac{5}{12} f\left(t_{n}, y_{n}\right)-\frac{16}{12} f\left(t_{n+1}, y_{n+1}\right)+\frac{23}{12} f\left(t_{n+2}, y_{n+2}\right)\right) \text {. }
$$

(iii) The Adams-Moulton method which is consistent of order 2 takes the form

$$
-y_{n}+y_{n+1}=h\left(\frac{1}{2} f\left(t_{n}, y_{n}\right)+\frac{1}{2} f\left(t_{n+1}, y_{n+1}\right)\right) \text {. }
$$

(iv) The BDF method which is consistent of order 2 takes the form

$$
\frac{1}{2} y_{n}-2 y_{n+1}+\frac{3}{2} y_{n+2}=h f\left(t_{n+2}, y_{n+2}\right) \text {. }
$$

(v) The Milne-Simpson method takes the form

$$
-y_{n}+y_{n+2}=h\left(\frac{1}{3} f\left(t_{n}, y_{n}\right)+\frac{4}{3} f\left(t_{n+1}, y_{n+1}\right)+\frac{1}{3} f\left(t_{n+2}, y_{n+2}\right)\right) \text {. }
$$

Example 1. Consider the pantograph equations [36]: 


$$
\left\{\begin{array}{l}
y_{1}^{\prime}(t)=-2.8 y_{1}(t)+3.6 y_{2}(t)-0.4 \sin (0.5 t) y_{1}(0.5 t)+0.8 \sin (0.5 t) y_{2}(0.5 t)+1+10 \sin t, \quad 0 \leq t \leq 1, \\
y_{2}^{\prime}(t)=3.6 y_{1}(t)-8.2 y_{2}(t)+0.8 \sin (0.5 t) y_{1}(0.5 t)-1.6 \sin (0.5 t) y_{2}(0.5 t)-2-20 \sin t, \quad 0 \leq t \leq 1, \\
y_{1}(0)=2, y_{2}(0)=1,
\end{array}\right.
$$

whose unique true solution is $y_{1}(t)=\sin t+2 e^{-t}, y_{2}(t)=-2 \sin t+e^{-t}$. Equation (81) is a special case $\in \mathbb{D}\left(L_{1}, L_{2}\right)$, where $L_{1}=10$ and $L_{2}=2 \sin 0.5 \approx 0.9588$ [36]. We use the methods $\mathrm{AB} 2, \mathrm{AM} 2$, and $\mathrm{B} 2$ with $h=\left(1 /\left(80 \times 2^{i}\right)\right)(i=0,1, \ldots, 5)$ to solve (81), respectively. The maximum global errors $E(h)$ and the observing orders $\widetilde{p}$ are listed in Table 1 .
From Table 1, we can see for the pantograph equation (81) the observing orders of the three methods AB2, AM2, and $\mathrm{B} 2$ are all very close to the theoretical convergent order 2.

Example 2. Consider the nonlinear VIDEs [46]:

$$
\left\{\begin{array}{l}
y_{1}^{\prime}(t)=2 y_{2}(t)-\frac{1}{3} t^{4}+\cos \left(y_{1}(t)\right)-1+\int_{0}^{t}\left(2 s \sin \left(y_{1}(s)\right)+s t y_{2}(s)\right) \mathrm{d} s, \quad 0 \leq t \leq 1 \\
y_{2}^{\prime}(t)=1-t \sin \left(y_{2}(t)\right)-\frac{1}{2} t^{2} \sin \left(y_{1}(t)\right)+\int_{0}^{t}\left(s t^{2} \cos \left(y_{1}(s)\right)+t \cos \left(y_{2}(s)\right)\right) \mathrm{d} s, \quad 0 \leq t \leq 1, \\
y_{1}(0)=0, y_{2}(0)=0 .
\end{array}\right.
$$

The unique true solution of equation (82) is $y_{1}(t)=t^{2}$ and $y_{2}(t)=t$. Let

$$
\begin{aligned}
y(t) & =\left[y_{1}(t), y_{2}(t)\right]^{T}, \\
f(t, u, \psi(\cdot)) & =\left[\begin{array}{c}
2 u_{2}-\frac{1}{3} t^{4}+\cos u_{1}-1+\int_{0}^{t}\left(2 s \sin \left(\psi_{1}(s)\right)+s t \psi_{2}(s)\right) \mathrm{d} s \\
1-t \sin u_{2}-\frac{1}{2} t^{2} \sin u_{1}+\int_{0}^{t}\left(s t^{2} \cos \left(\psi_{1}(s)\right)+t \cos \left(\psi_{2}(s)\right)\right) \mathrm{d} s
\end{array}\right],
\end{aligned}
$$

where $0 \leq t \leq 1, u=\left[u_{1}, u_{2}\right]^{T} \in \mathbf{R}^{2}$, and $\psi \in \mathbf{C}_{2}[0,1]$. Then, equation (82) can be written in form $(1) \in \mathbb{D}\left(L_{1}, L_{2}\right)$, where $L_{1}$ and $L_{2}$ are both of moderate size. We use the method AB2 with $h=\left(1 /\left(80 \times 2^{i}\right)\right)(i=0,1, \ldots, 6)$ to solve (82), respectively. The maximum global errors $E(h)$ and the observing orders $\tilde{p}$ are listed in Table 2 .
From Table 2, we can see, for the nonlinear VIDEs (82), the observing orders of the method $\mathrm{AB} 2$ are all very close to the theoretical convergent order 2 .

Example 3. Consider the nonlinear VDIDEs [36]:

$$
\left\{\begin{array}{cl}
y^{\prime}(t)=y^{2}(t)+2 y(t)-2 y\left(t-\frac{\pi}{2}\right)+2 y\left(\frac{t}{2}\right) y\left(\frac{t-\pi}{2}\right)+\int_{t-\pi}^{t-(\pi / 2)} y(z) \mathrm{d} z+\cos ^{2} t-1, & 0 \leq t \leq 6 \\
y(t)=\sin t, & -\pi \leq t \leq 0 .
\end{array}\right.
$$

The unique true solution of equation (84) is $y(t)=\sin t$. Equation $(84) \in \mathbb{D}\left(L_{1}, L_{2}\right)$ in the sense of Remark 6, where $L_{1}$ and $L_{2}$ are both of moderate size [36]. We use MS4 with $h=\left(0.01 / 2^{i}\right)(i=0,1,2,3)$ to solve (84), respectively, and list the maximum global errors $E(h)$ and the observing orders $\tilde{p}$ in Table 3. 
TABLE 1: The maximum global errors $E(h)$ and the observing orders $\widetilde{p}$ produced by AB2, AM2, and B2 when applied to equation (81).

\begin{tabular}{|c|c|c|c|c|c|c|}
\hline \multirow{2}{*}{$h$} & \multicolumn{2}{|c|}{$\mathrm{AB} 2$} & \multicolumn{2}{|c|}{ AM2 } & \multicolumn{2}{|c|}{ B2 } \\
\hline & $E(h)$ & $\widetilde{p}$ & $E(h)$ & $\tilde{p}$ & $E(h)$ & $\widetilde{p}$ \\
\hline $1 / 80$ & $5.179403 \times 10^{-5}$ & & $9.921322 \times 10^{-6}$ & & $4.066459 \times 10^{-5}$ & \\
\hline $1 / 160$ & $1.297365 \times 10^{-5}$ & 1.997201 & $2.497546 \times 10^{-6}$ & 1.990021 & $1.021635 \times 10^{-5}$ & 1.992893 \\
\hline $1 / 320$ & $3.246682 \times 10^{-6}$ & 1.998546 & $6.265474 \times 10^{-7}$ & 1.995016 & $2.560343 \times 10^{-6}$ & 1.996471 \\
\hline $1 / 640$ & $8.120817 \times 10^{-7}$ & 1.999269 & $1.569068 \times 10^{-7}$ & 1.997516 & $6.408666 \times 10^{-7}$ & 1.998241 \\
\hline $1 / 1280$ & $2.030719 \times 10^{-7}$ & 1.999634 & $3.926050 \times 10^{-8}$ & 1.998757 & $1.603144 \times 10^{-7}$ & 1.999120 \\
\hline $1 / 2560$ & $5.077445 \times 10^{-8}$ & 1.999816 & $9.819351 \times 10^{-9}$ & 1.999379 & $4.009081 \times 10^{-8}$ & 1.999561 \\
\hline
\end{tabular}

TABle 2: The maximum global errors $E(h)$ and the observing orders $\widetilde{p}$ produced by $\mathrm{AB} 2$ when applied to equation (82).

\begin{tabular}{lcc}
\hline$h$ & $E(h)$ & $\tilde{p}$ \\
\hline $1 / 80$ & $2.567537 \times 10^{-6}$ & \\
$1 / 160$ & $6.421382 \times 10^{-7}$ & 1.999429 \\
$1 / 320$ & $1.605505 \times 10^{-7}$ & 1.999857 \\
$1 / 640$ & $4.013861 \times 10^{-8}$ & 1.999965 \\
$1 / 1280$ & $1.003472 \times 10^{-8}$ & 1.999990 \\
$1 / 2560$ & $2.508682 \times 10^{-9}$ & 1.999999 \\
$1 / 5120$ & $6.271714 \times 10^{-10}$ & 1.999998 \\
\hline
\end{tabular}

TABle 3: The maximum global errors $E(h)$ and the observing orders $\widetilde{p}$ produced by MS4 when applied to equation (84).

\begin{tabular}{lcc}
\hline$h$ & $E(h)$ & $\widetilde{p}$ \\
\hline 0.01 & $6.757335 \times 10^{-7}$ & \\
0.005 & $4.041475 \times 10^{-8}$ & 4.063501 \\
0.0025 & $2.483531 \times 10^{-9}$ & 4.024417 \\
0.00125 & $1.524486 \times 10^{-10}$ & 4.025998 \\
\hline
\end{tabular}

From Table 3, we can see, for the nonlinear VDIDEs (84), the observing orders of the method MS4 are all very close to the theoretical convergent order 4 .

\section{Data Availability}

The data used to support the findings of this study are available from the corresponding author upon request.

\section{Conflicts of Interest}

The authors declare there are no conflicts of interest.

\section{Acknowledgments}

This work was supported by Hunan Provincial Natural Science Foundation of China (Grant no. 2017JJ2194), the Scientific Research Fund of Hunan Provincial Education Department (Grant no. 20C1261), and the PhD Start-Up Fund from Hunan University of Arts and Science (Grant no. 16BSQD24).

\section{References}

[1] J. K. Hale and S. M. Verduyn Lunel, Introduction to Functional Differential Equations, Springer, Berlin, Germany, 1993.

[2] Y. Kuang, Delay Differential Equations with Applications in Population Dynamics, Academic Press, Cambridge, UK, 1993.
[3] C. T. H. Baker, G. A. Bocharov, and F. A. Rihan, "A report on the use of delay differential equations in numerical modelling in the biosciences," Technical Report, MCCM, Kaniyur, India, 1999.

[4] G. A. Bocharov and F. A. Rihan, "Numerical modelling in biosciences using delay differential equations," Journal of Computational and Applied Mathematics, vol. 125, no. 1-2, pp. 183-199, 2000.

[5] J. P. Richard, "Time-delay systems: an overview of some recent advances and open problems," Automatica, vol. 39, no. 10, pp. 1667-1694, 2003.

[6] L. Torelli, "Stability of numerical methods for delay differential equations," Journal of Computational and Applied Mathematics, vol. 25, no. 1, pp. 15-26, 1989.

[7] A. Bellen and M. Zennaro, "Strong contractivity properties of numerical methods for ordinary and delay differential equations," Applied Numerical Mathematics, vol. 9, no. 3-5, pp. 321-346, 1992.

[8] K. J. In't Hout, “A new interpolation procedure for adapting Runge-Kutta methods to delay differential equations," BIT, vol. 32, pp. 634-649, 1992.

[9] G.-D. Hu and G.-D. Hu, "Stability of Runge-Kutta methods for delay differential systems with multiple delays," IMA Journal of Numerical Analysis, vol. 19, no. 3, pp. 349-356, 1999.

[10] A. Bellen and M. Zennaro, Numerical Methods for Delay Differential Equations, Oxford University Press, Oxford, UK, 2003.

[11] H. Tian and Q. Guo, "Dynamics of linear multistep methods for delay differential equations," International Journal of Bifurcation and Chaos, vol. 14, no. 1, pp. 329-336, 2004.

[12] J. X. Kuang and Y. H. Cong, Stability of Numerical Methods for Delay Differential Equations, Science Press, Beijing, China, 2005.

[13] C. Huang and S. Vandewalle, "Discretized stability and error growth of the nonautonomous pantograph equation," SIAM Journal on Numerical Analysis, vol. 42, no. 5, pp. 2020-2042, 2005.

[14] L. P. Wen and S. F. Li, "Stability of theoretical solution and numerical solution of nonlinear differential equations with piecewise delays," Journal of Computational Mathematics, vol. 23, no. 4, pp. 393-400, 2005.

[15] M. Z. Liu, Z. W. Yang, and G. D. Hu, "Asymptotical stability of numerical methods with constant stepsize for pantograph equations," BIT Numerical Mathematics, vol. 45, no. 4, pp. 743-759, 2005.

[16] S. Gan, "Dissipativity of $\theta$-methods for nonlinear delay differential equations of neutral type," Applied Numerical Mathematics, vol. 59, no. 6, pp. 1354-1365, 2009.

[17] S. Sedaghat, Y. Ordokhani, and M. Dehghan, "Numerical solution of the delay differential equations of pantograph type via Chebyshev polynomials," Communications in Nonlinear 
Science and Numerical Simulation, vol. 17, no. 12, pp. 48154830, 2012.

[18] G. Zhang and A. Xiao, "Stability and convergence analysis of implicit-explicit one-leg methods for stiff delay differential equations," International Journal of Computer Mathematics, vol. 93, no. 11, pp. 1964-1983, 2016.

[19] W. Wang, "Uniform ultimate boundedness of numerical solutions to nonlinear neutral delay differential equations," Journal of Computational and Applied Mathematics, vol. 309, pp. 132-144, 2017.

[20] C. T. H. Baker, A. Makroglou, and E. Short, "Regions of stability in the numerical treatment of Volterra integro-differential equations," SIAM Journal on Numerical Analysis, vol. 16, no. 6, pp. 890-910, 1979.

[21] H. Brunner, "Implicit Runge-Kutta methods of optimal order for Volterra integro-differential equations," Mathematics of Computation, vol. 42, no. 165, pp. 95-109, 1984.

[22] D. Xu, "Stability of the difference type methods for linear Volterra equations in Hilbert spaces," Numerische Mathematik, vol. 109, pp. 571-595, 2008.

[23] M. Shakourifar and M. Dehghan, "On the numerical solution of nonlinear systems of Volterra integro-differential equations with delay arguments," Computing, vol. 82, no. 4, pp. 241-260, 2008.

[24] T. Koto, "Stability of Runge-Kutta methods for delay integrodifferential equations," Journal of Computational and Applied Mathematics, vol. 145, no. 2, pp. 483-492, 2002.

[25] C. Zhang and S. Vandewalle, "General linear methods for Volterra integro-differential equations with memory," SIAM Journal on Scientific Computing, vol. 27, no. 6, pp. 2010-2031, 2006.

[26] C. Huang and S. Vandewalle, "Stability of Runge-Kutta-Pouzet methods for Volterra integro-differential equations with delays," Frontiers of Mathematics in China, vol. 4, no. 1, pp. 63-87, 2009.

[27] H. Chen and C. Zhang, "Convergence and stability of extended block boundary value methods for Volterra delay integro-differential equations," Applied Numerical Mathematics, vol. 62, no. 2, pp. 141-154, 2012.

[28] S. Sedaghat, Y. Ordokhani, and M. Dehghan, "On spectral method for Volterra functional integro-differential equations of neutral type," Numerical Functional Analysis and Optimization, vol. 35, no. 2, pp. 223-239, 2014.

[29] Y. Cong, Y. Wang, and G. Hu, "Weak delay-dependent stability of Runge-Kutta methods for differential systems with distributed delays," Journal of Computational and Applied Mathematics, vol. 342, pp. 70-82, 2018.

[30] S. Li, "B-theory of Runge-Kutta methods for stiff Volterra functional differential equations," Science in China Series A, vol. 46, no. 5, pp. 662-674, 2003.

[31] S. Li, "Stability analysis of solutions to nonlinear stiff Volterra functional differential equations in Banach spaces," Science in China Series A, vol. 48, no. 3, pp. 372-387, 2005.

[32] S. Li, "B-theory of general linear methods for Volterra functional differential equations," Applied Numerical Mathematics, vol. 53, no. 1, pp. 57-72, 2005.

[33] S. Li, "A review of theoretical and numerical analysis for nonlinear stiff Volterra functional differential equations," Frontiers of Mathematics in China, vol. 4, no. 1, pp. 23-48, 2009.

[34] S. Li, "High order contractive Runge-Kutta methods for Volterra functional differential equations," SIAM Journal on Numerical Analysis, vol. 47, no. 6, pp. 4290-4325, 2010.
[35] S. F. Li, Numerical Analysis for Stiff Ordinary and Functional Differential Equations, Xiangtan University Press, Xiangtan, China, (in Chinese), 2010.

[36] S. F. Li, "Classical theory of Runge-Kutta methods for Volterra functional differential equations," Applied Mathematics and Computation, vol. 230, pp. 78-95, 2014.

[37] R. D. Driver, "Existence and stability of solutions of a delaydifferential system," Archive for Rational Mechanics and Analysis, vol. 10, no. 1, pp. 401-426, 1962.

[38] P. Henrici, Discrete Variable Methods in Ordinary Differential Equations, John Wiley \& Sons, Hoboken, NJ, USA, 1962.

[39] J. D. Lambert, Computational Methods in Ordinary Differential Equations, John Wiley \& Sons, Hoboken, NJ, USA, 1973.

[40] G. Dahlquist, "Convergence and stability in the numerical integration of ordinary differential equations," Mathematica Scandinavica, vol. 4, pp. 33-53, 1956.

[41] K. Dekker and J. G. Verwer, Stability of Runge-Kutta Methods for Stiff Nonlinear Differential Equations, North Holland, Amsterdam, The Netherlands, 1984.

[42] F. Bashforth and J. C. Adams, Theories of Capillary Action, Cambridge University Press, Cambridge, UK, 1883.

[43] F. R. Moulton, New Methods in Exterior Ballistics, University Chicago Press, 1926.

[44] W. E. Milne, "Numerical integration of ordinary differential equations," The American Mathematical Monthly, vol. 33, no. 9, pp. 455-460, 1926.

[45] W. E. Milne, Numerical Solution of Differential Equations, John Wiley \& Sons, Hoboken, NJ, USA, 1953.

[46] M. I. Berenguer, A. I. Garralda-Guillem, and M. Ruiz Galán, "An approximation method for solving systems of Volterra integro-differential equations," Applied Numerical Mathematics, vol. 67, pp. 126-135, 2013. 